\title{
Infants' Social Evaluation of Helpers and Hinderers: A Large-Scale, Multi-Lab, Coordinated Replication Study
}

The ManyBabies Consortium, consisting of:

Kelsey Lucca (Arizona State University)*

Krista Byers-Heinlein (Concordia University)

Arthur Capelier-Mourguy (Lancaster University)

Laura Cirelli (University of Toronto)

Rodrigo Dal Ben (Concordia University)

Michael C. Frank (Stanford University)

Annette M. E. Henderson (University of Auckland)

Jonathan F. Kominsky (Rutgers University)

Zoe Liberman (University of California, Santa Barbara)

Francesco Margoni (University of Oslo)

Peter Reschke (Brigham Young University)

Laura Schlingloff (Central European University)

Kim Scott (Massachusetts Institute of Technology)

Melanie Soderstrom (University of Manitoba)

Jessica Sommerville (University of Toronto)

Yanjie Su (Peking University)

Denis Tatone (Central European University)

Florina Uzefovsky (Ben-Gurion University of the Negev)

Yiyi Wang (Peking University)

Francis Yuen (University of British Columbia)

J. Kiley Hamlin (University of British Columbia)

\section{ADDITIONAL AUTHORS WILL BE ADDED IN ALPHABETICAL ORDER AFTER DATA} COLLECTION

*Corresponding author klucca@asu.edu

Acknowledgements: We would like to thank the many families who participated in this research.

We would also like to thank all of the researchers who participated in planning this study as well as those researchers who participated in pilot testing including Siobhan Kennedy-Costantini. This research was supported in part by XXXX awarded to XXX; a grant from NICHD (1R01HD076949-01) awarded to Jessica Sommerville; a Social Sciences and Humanities Research Council of Canada (SSHRC) grant (12R20573) awarded to J. Kiley Hamlin, an NSERC Discovery Grant RGPIN-05367-2019 awarded to Melanie Soderstrom, an EU Horizon 2020 ERC Advanced Grant (742231) awarded to Gergely Csibra, a University of Auckland PBRF grant awarded to Annette Henderson. The opinions expressed in this publication are those of the authors and do not necessarily reflect the views of the funding agencies listed here. The authors declare no conflicts of interest.

Data availability statement: The data that support the findings of this study will be openly available on GitHub at https://github.com/manybabies/mb4-analysis. 
INFANTS' SOCIAL EVALUATIONS

Research Highlights (to be completed after data collection)

- The current study examined infants' preferences for prosocial (helping) over antisocial (hindering) individuals through a large-scale, multi-lab, coordinated replication study.

- Research highlight describing final sample.

- Research highlight briefly summarizing findings.

- Research highlight discussing implications of findings. 
INFANTS' SOCIAL EVALUATIONS

\begin{abstract}
Evaluating others' actions as praiseworthy or blameworthy is a fundamental aspect of human nature. A seminal study published in 2007 suggested that the ability to form social evaluations based on third-party interactions emerges within the first year of life, considerably earlier than previously thought (Hamlin, Wynn, \& Bloom, 2007). In this study, infants demonstrated a preference for a character (i.e., a shape with eyes) who helped, over one who hindered, another character who tried but failed to climb a hill. This study sparked a new line of inquiry into infants' social evaluations; however, numerous attempts to replicate the original findings yielded mixed results, with some reporting effects not reliably different from chance. These failed replications point to at least two possibilities: (1) the original study may have overestimated the true effect size of infants' preference for helpers, or (2) key methodological or contextual differences from the original study may have compromised the replication attempts. Here we present a pre-registered, closely coordinated, multi-laboratory, standardized study aimed at replicating the helping/hindering finding using a well-controlled video version of the hill show. We intended to (1) provide a precise estimate of the true effect size of infants' preference for helpers over hinderers, and (2) determine the degree to which infants' preferences are based on social features of the Helper/Hinderer scenarios. XYZ labs participated in the study yielding a total sample size of XYZ infants between the ages of 5.5 and 10.5 months. BRIEF SUMMARY OF RESULTS.
\end{abstract}

Keywords: moral development; social cognition; infancy; reproducibility; experimental methods; social development 
INFANTS' SOCIAL EVALUATIONS

\section{Introduction}

As adults, we are quick to judge other individuals' actions as praiseworthy or blameworthy. These judgments have a pervasive impact on our social interactions: we gravitate toward and befriend individuals with a history of behaving prosocially, and avoid those with a history of behaving antisocially. Notably, our judgments and selective social preferences are not limited to those with whom we directly interact. Humans readily judge individuals on the basis of the prosocial and antisocial actions they direct toward unrelated third parties, and even incur personal costs to punish those who behave antisocially (Fehr \& Fischbacher, 2003). From where does this propensity to morally evaluate others originate?

Historically, some scholars have contended that infants are born either amoral, with no moral sense, or immoral, motivated solely by selfish impulses (Freud, 1961; Kohlberg, 1969; Piaget, 1932). According to these perspectives, it is only through cognitive development and extensive socialization that humans come to develop an adult-like moral sense (reviewed in Brownell, 2013; Carpendale \& Lewis, 2004). This proposal has been recently challenged by a series of studies on the social evaluative abilities of preverbal infants (reviewed in Margoni \& Surian, 2018). This work suggests that key precursors of full-fledged moral competencies, such as the ability to evaluate others on the basis of their prosocial or antisocial acts, may already be in place within the first year after birth.

The first study to suggest that preverbal infants engage in social evaluations presented 6and 10-month-old infants with scenarios in which novel characters directed prosocial and antisocial acts toward a third party (Hamlin et al., 2007). Specifically, infants watched a puppet show featuring a "Climber" (a red wooden circle with googly eyes) who repeatedly tried but failed to climb to the top of a steep hill. Infants were shown two distinct events in alternation: a 


\section{INFANTS' SOCIAL EVALUATIONS}

hindering event and a helping event. During hindering events, a Hinderer character (e.g., a blue wooden square with googly eyes) prevented the Climber from reaching its goal by pushing it down to the bottom of the hill. During helping events, a Helper character (e.g., a yellow wooden triangle with googly eyes; character identity was counterbalanced across infants) facilitated the Climber's goal by pushing it to the top of the hill. Helping and Hindering events were repeated until infants reached a pre-set habituation criterion. Finally, infants were presented with the Helper and the Hinderer and were prompted to choose between the two. Infants at both 6 and 10 months of age robustly reached for the Helper over the Hinderer (12 of 12 6-month-olds and 14 of 16 10-month-olds), suggestive of early social evaluation.

A key control condition tested whether preferences for the Helper were truly social in nature or whether they were based on low-level features of the display. In this control condition, the procedure was similar to the experimental condition, except that the animate Climber was replaced with an inanimate (eyeless) red circular ball that produced no self-propelled motion. On alternating events, this ball was pushed up or down the hill by the same square and triangle characters that played the role of Helper and Hinderer in the original condition. Critically, in this condition pushing up and down was present, but should not have been interpreted as helping and hindering, given that the ball did not exhibit cues of agency or goal-directedness. Here, infants did not demonstrate a preference for either character, suggesting that infants' evaluations of the Helper and Hinderer in the experimental condition were guided by the social consequences of their actions (Hamlin et al., 2007).

In recent years, research has extended these findings across a variety of paradigms and social scenarios. This work demonstrated that infants, as early as five months, prefer characters who helped, rather than hindered, an agent achieve different types of goals, including opening a 


\section{INFANTS' SOCIAL EVALUATIONS}

box containing an appealing toy, retrieving a toy from a high shelf, and obtaining a preferred object (Hamlin \& Wynn, 2011; Hamlin et al., 2013; Woo, Steckler, Le, \& Hamlin, 2017). Infants' preference for prosocial individuals has also been shown to extend to morally relevant actions beyond helping and hindering; for example, 6-10-month-olds disprefer those who physically batter others but prefer those who prevent others from being battered (Kanakogi et al., 2013; 2017; see also Buon et al., 2014), and by 13-16 months, infants demonstrate a preference for agents who act fairly versus unfairly (e.g., distributed resources equally versus unequally; Burns \& Sommerville, 2014; Geraci \& Surian, 2011; Lucca, Pospisil, \& Sommerville, 2018). As in the original Helper/Hinderer study, control conditions have often helped to rule out various low-level explanations for infants' preferences, suggesting that they were based on the social nature of the interactions.

Findings suggesting that infants possess precocious social evaluation capacities have led researchers to probe the replicability of these results, as well as the underlying explanation for infants' success in these tasks. Direct replication attempts have been met with varying levels of success: Though some studies have found that infants prefer prosocial individuals in manual choice and preferential looking paradigms (Buon et al., 2014, with 10- and 29-month-olds; Chae \& Song, 2018, with 6- and 10-month-olds; Hamlin et al., 2010, with 3-month-olds; LoheideNiesmann et al., 2020, with 24-month-olds; Scola et al., 2015; with 12-24 and 24-36-month-olds; Shimizu et al., 2018, with 15-18-month-olds), others have not (i.e. Abramson et al., unpublished, with 9- and 18-month-olds; Cowell \& Decety, 2015, with 12- to 24-month-olds; Nighbor et al., 2017, with 5- to 16-month-olds; Salvadori et al., 2015, with 9-month-olds; Schlingloff et al., 2020, with 14- to 16-month-olds; Shimizu et al., 2018, with 6-, 9-, and 12-month-olds; Vaporova \& Zmyj, 2020, with 9- and 14-month-olds). These disparate findings have raised critical 


\section{INFANTS' SOCIAL EVALUATIONS}

questions about the robustness of the effect, as well as the conditions under which the effect can be elicited.

Other research has focused on identifying the reason for infants' choices in social evaluation tasks. Most notably, Scarf and colleagues (2012) raised the possibility that infants' preference for helping characters could be explained not by a preference for helpful over unhelpful agents, but by perceptual features that co-occurred with the helping action in Hamlin and colleagues' (2007) experiment. Specifically, they noted that the climbing character bounced after being helped, but not after being hindered. Thus, infants may have selected the Helper solely due to its association with a positively valenced stimulus (i.e., the bouncing event). To examine this possibility, Scarf and colleagues conducted a series of experiments with 10-monthold infants that closely matched the original study, but with additional conditions in which bouncing actions also occurred during hindering events. Results revealed that infants preferred characters associated with bouncing, regardless of the type of event (helping or hindering) in which the action occurred. These results suggested that infants' evaluations may be based on physical rather than social aspects of the displays. Subsequent research challenged this interpretation (cf. Hamlin, 2015), noting that stimuli differences may have hindered infants' ability to represent the Climber's behavior as goal-directed. Nevertheless, the study by Scarf and colleagues (2012) highlights that infants' evaluations in these tasks could be based on features of the events devoid of sociomoral significance thereby confirming the necessity of proper control conditions in studies claiming to examine the roots of social evaluation in infancy.

A recent meta-analysis aimed to provide an estimate of the effect size of infants' preference (or lack thereof) for prosocial over antisocial characters, as well as to provide insights into potential moderators of the effect (Margoni \& Surian, 2018; see also Holvoet, Scola, 


\section{INFANTS' SOCIAL EVALUATIONS}

Arciszewski, \& Picard, 2016). The meta-analysis included data from 26 studies (reporting a total of 61 effect sizes) in which a prosocial agent was defined in various ways, including helping (versus hindering), giving (versus taking), or distributing goods fairly (versus unfairly), and in which preference was defined either by selective reaching or selective helping. Overall, the estimated average proportion of infants who preferred the prosocial character was $.68,95 \% \mathrm{CI}$ $[0.64,0.72]$.

The overall effect size did not vary as a function of age (4 to 32 months), the type of dependent variable (reaching versus helping), target type (foam shapes, hand puppets, or human experimenters), or type of stimulus presentation (real events or video displays). In contrast, infants' preference for prosocial characters did depend on the type of action presented: Studies depicting giving versus taking yielded larger effect sizes than did studies depicting helping versus hindering (although this finding should be interpreted with caution due to the small number of datapoints for giving vs. taking). The authors also noted higher effect sizes in studies with small sample sizes ( $N=16$ or fewer), suggestive of a file-drawer problem, as well as in the laboratories that produced the original studies on the topic (e.g., Hamlin's), indicative of a lab effect. Finally, they explored for evidence of a publication bias. Although most of the examined effects were published $(N=44)$, several were unpublished $(N=17)$. A common "trim-and-fill" procedure (Duval \& Tweedie, 2000) provided evidence of a publication bias (but see Carter, Schönbrodt, Gervais, \& Hilgard, 2019). When adjusted for publication bias, the meta-analytic estimate was slightly smaller, revealing an average proportion of choice for the Helper character of .64, 95\% CI [0.60, 0.69].

The results of the meta-analysis raised several important directions for future work. Since nearly half of the studies included in the meta-analysis were conducted by a single lab using 


\section{INFANTS' SOCIAL EVALUATIONS}

small sample sizes $(N=16$ infants per study), replication studies that incorporate a higher diversity of labs as well as larger samples are clearly needed. Moreover, some of the studies incorporated in the meta-analysis were failed replications in which no preference for prosocial individuals was observed. These failures suggest either that the true effect size might be smaller than originally thought (or non-existent), and/or that slight variations in method or population were responsible for the failures (Makel, Plucker, \& Hegarty, 2012). Moreover, variability between studies can also be partially explained by sampling error or chance alone (see Margoni \& Shepperd, 2020; Stanley \& Spencer, 2014). Finally, the meta-analysis did not examine infants' behavior in control conditions, leaving open questions about whether infants' preferences are truly based on the social aspects of prosocial and antisocial actions.

The current study set out to address these outstanding questions as well as provide new insights into infants' early social evaluations. Specifically, we first aimed to establish a precise estimate of the true effect size of infants' preference for helping over hindering agents. Second, we sought to determine whether infants' preferences are social in nature; namely that they require that positive and negative acts be directed toward agentic third parties capable of goaldirected behavior. To achieve these objectives, we conducted a large-scale, multi-site replication study of infants' preferences for helping characters, with a pre-registered methodological and analytical plan [preregistration will be posted to OSF after an "in principle acceptance" is received]. Our multi-site replication approach provides crucial insights beyond those of the existing systematic reviews (Holvoet et al., 2016; Margoni \& Surian, 2018). First, by using a consistent methodology across laboratories around the world, we are able to more precisely identify sources of variation in infants' social evaluations (e.g., age, geographic location) that go beyond variation in the stimuli and experimental procedure, since each lab in our study will 


\section{INFANTS' SOCIAL EVALUATIONS}

follow the same protocol and use the same video stimuli. Second, this approach allows us to compare infants' preferences for characters across social and non-social contexts, enabling us to measure whether infants' preferences are driven by social features of the helping/hindering events versus non-social or perceptual aspects of the events. Finally, given that meta-analyses in psychology have been shown to report effect sizes approximately three times as large as preregistered multi-lab replication projects (e.g. due to publication bias or selective reporting), our approach will allow us to obtain a more accurate estimate of the true effect size of infants' preference for Helpers (Kvarven, Strømland, \& Johannesson, 2020).

We utilized the ManyBabies model of coordinated replication efforts (Byers-Heinlein et al., 2020; Frank et al., 2017; ManyBabies Consortium, 2020; see also ManyLabs: Klein et al., 2014), wherein a hypothesis of interest is chosen and explored by a large group of interested laboratories, all following a standardized protocol in order to create a data set larger than any one laboratory could produce on its own. This method allows for the exploration of both participantand laboratory-level variables. The ManyBabies model strives to adhere to Open Science principles and practices. For instance, all stimuli, protocols, code, and data are shared on open access repositories. As opposed to exactly reproducing previously published methodologies, group-level decision making is used in order to converge on a method that provides the best possible test of a hypothesis of interest. Laboratories from across the globe, especially from countries that are traditionally underrepresented in developmental research, are invited to contribute at all research stages. Whereas these collective efforts focus on carefully deciding and standardizing a study's critical manipulations, individual labs still utilize their own general research practices (e.g., recruitment strategies, research assistant training). 


\section{INFANTS' SOCIAL EVALUATIONS}

To examine whether infants do indeed engage in social evaluation of prosocial and antisocial characters, we chose to replicate the hill study by Hamlin and colleagues (2007). This study was selected because: (1) it is the most widely cited demonstration of infant social evaluation in the literature, (2) it has been successfully replicated in subsequent research by the original lab (Hamlin, Wynn, \& Bloom, 2010) and at least one independent laboratory (Chae \& Song, 2018), but not by other others (Cowell \& Decety, 2015; Scarf et al., 2012; Schlingloff et al., 2020); (3) the effect has been reported in studies employing multiple response measures (including preferential looking, anticipatory looking, and selective reaching) and different presentation formats (video stimuli presented on screen rather than live displays: Hamlin, 2015). We reasoned that video stimuli would be easier to utilize on a global scale.

Consistent with the ManyBabies goal of conducting the best possible test of a given hypothesis as opposed to exact replications, we made several modifications to the original Hamlin and colleagues (2007) paradigm. First, as just noted, we utilized a filmed puppet show as opposed to a live puppet show as in the original study. Using pre-recorded stimuli standardizes the stimuli presented to infants across laboratories, thereby ensuring that any differences in results can not be attributed to variations in habituation events. Further, videotaping these events rather than producing them in real-time allowed us to, within condition, match the speed and timing of the pushing-up and down actions along with the overall exposure to the push-up/pushdown characters down to the millisecond. This method of presentation also increases the number of labs eligible for participation because it substantially reduces barriers to participation, such as financial/time constraints involved in purchasing puppet stage materials, constructing a puppet stage, and training researchers to execute a live puppet show. The video stimuli used here were recorded in Hamlin's lab and closely matched videos used in a successful replication (Hamlin, 


\section{INFANTS' SOCIAL EVALUATIONS}

2015) of the live puppet show paradigm (Hamlin et al., 2007). Because these two studies (Hamlin, 2015; Hamlin et al., 2007) differed only in the stimulus presentation modality and found results with comparable effect sizes, we expected that the effect size would not be moderated by this decision. Importantly, meta-analytic results also found no moderating effect of the modality of stimulus presentation (i.e., animations, videotaped, and real events; Margoni \& Surian, 2018).

Second, our design implemented controls for perceptual differences between helping and hindering events that were not present in the initial Hamlin and colleagues (2007) study. As previously discussed, Scarf and colleagues (2012) argued that infants' preferences in the hill paradigm were due to the Climber character bouncing after being helped but not after being hindered (but see Hamlin, 2015, for evidence against these criticisms). To avoid this issue, our study utilized videos in which the Climber remained motionless, instead of bouncing, upon reaching its final position.

Third, rather than including two separate age groups (6- and 10-month-olds), as in the original study, we included a single group of infants ranging from 5.5 months to 10.5 months. This age range was selected for several reasons. First, a manual reaching choice task can be used across this age window, allowing us to fully standardize the task across all infants. Second, infants in this age range demonstrate sensitivity to the causal power of agents (Liu et al., 2019), and to both successful (e.g., Woodward, 1998) and failed goal-directed actions (e.g., Brandone \& Wellman, 2009; Hamlin, Hallinan, \& Woodward, 2008). Third, although Margoni and Surian (2018) did not find a significant influence of age on infants' preference for prosocial individuals, several successful and failed replications fall within this age range (Hamlin, 2015; Hamlin et al 2011; Salvadori et al., 2015; Scarf et al., 2012). Thus, including this broad age range allowed us 


\section{INFANTS' SOCIAL EVALUATIONS}

to assess whether there are developmental changes in infants' preferences for prosocial others. Finally, as recruiting participants across a broad age range is presumably easier than recruiting within a narrow age range, we selected a wide age window to maximize the number of laboratories able to participate.

As in the original Hamlin and colleagues (2007) study, we included a Non-Social control condition to examine whether infants' preferences are driven by the social aspects of helping versus hindering actions as opposed to non-social perceptual features of the displays. In the control condition, infants viewed events similar to the helping and hindering events of the Social condition, but with several notable differences. Most critically, the Climber was replaced by an inanimate, eyeless object that did not engage in self-propelled motion. Specifically, infants viewed an inert red ball being pushed up or down the hill by triangle and square agentic characters with eyes. Based on the estimate from Margoni and Surian's (2018) meta-analysis, we predicted that approximately $64 \%$ of infants would choose the Helper in the Social condition where the animate climber, a red ball with eyes, demonstrated an unfulfilled goal to climb the hill. We predicted that infants would not demonstrate a preference for the character who pushed an inanimate red ball (that had no eyes and demonstrated no goal-directed behavior) in the NonSocial control condition. Relatedly, we also predicted significantly greater preference for the Helper in the Social compared to the pusher-upper in the Non-Social condition.

The Social and Non-Social videos were designed to convey fundamentally different events -- helping/hindering an animate character versus pushing an inanimate character up/down -- therefore, it was necessary that they differed in several ways. First, we had to ensure that the ball was perceived as animate in the Social videos, and as inanimate in the Non-Social videos. To do so, Social videos included a hill-climbing action at the start which demonstrated the ball's 


\section{INFANTS' SOCIAL EVALUATIONS}

goal to go uphill. The Non-Social videos do not have this portion of the video, since the ball is inanimate and not capable of self-propelled motion. This difference led to the Non-Social videos being 4.4 seconds shorter than the Social videos. Although timing of the Non-Social videos could have been matched to the Social videos by introducing additional still frames and/or adding in novel actions, we reasoned that these modifications might lead to inattentiveness and fussiness in the Non-Social displays, insofar as they do not add anything directly relevant to, or may even hamper the interpretability of the push-up or push-down character's goals. Despite the overall length across Social and Non-Social videos, the length of the videos was nevertheless equated within condition (i.e., both Social videos are 13.3s and both Non-Social videos are 8.9 s), and the amount of time the helper/push-up and hinderer/push-down characters are on stage are exactly matched within and closely matched across conditions $($ Social $=4.7 \mathrm{~s}$, Non-Social $=$ $5.9 \mathrm{~s})$. Although we do not expect these differences in timing to impact our main results of interest, we will test for the possible influence of these timing differences by analyzing infants' attention (e.g., as measured by the number of habituation trials and the overall looking to the still frame events presented after each video), and explore whether (a) attention differs across conditions, and (b) differences in attention following each event relate to differences in infants' choices.

A second difference between the Social and Non-Social conditions involves the location of the climber at the start of each event. In the Social condition the climber always has the goal to climb up a steep hill, and needs assistance in doing so. Therefore, the climber starts at the bottom of the hill during both helping and hindering events (before being pushed up or down). In the Non-Social condition, infants also see the ball being pushed up or down the hill. But, because the ball is inanimate and not-goal directed, the ball must begin at the bottom of the hill during 


\section{INFANTS' SOCIAL EVALUATIONS}

pushing-up events, but at the top of the hill during pushing-down events. Although we could have created events where the character was trying to climb down a hill, we thought this goal might be perceived as relatively trivial in terms of costs (compared to climbing up a steep hill), rendering it more difficult to determine that the climber needs assistance. We have no reason to hypothesize that these differences in starting location across conditions would influence infants' choices.

Because the critical event across conditions is the pushing action itself, it was important that the mechanics of the pushing actions were as similar as possible across conditions so the characters that interacted with the ball would appear equally agentive and goal-directed. Both conditions featured two pushing actions, each of which began with a forceful "smack". In the Social condition, these smacks occur during the climbing action (i.e., on the steep part of the hill). In the Non-Social condition, on the other hand, the smacks occur on the flat portions of the hill. This difference across conditions is due to the physical properties of pushing objects: smacking an inanimate ball on the steep part of the hill should cause the ball to roll down on its own, rendering the second smack unnecessary. This difference in the location of the smacks required the characters in the Non-Social condition to stay in contact with the red circle for longer ( $\sim 3$ seconds) than the characters in the Social condition $(\sim 1$ second). Importantly, however, the contact time between characters within conditions is the same. Nevertheless, as with the differences in overall timing, we will conduct exploratory analyses to examine possible relations between infants' attention (e.g., as measured by looking-time to the freeze frames presented at the end of each video and the number of trials it took for the infants to habituate) and subsequent choices.

\section{Methods}


INFANTS' SOCIAL EVALUATIONS

Note that some methodological details may appear in online supplemental materials rather than in the main text.

\section{Participation Details}

Time-frame. We circulated an open call for lab participation after our Registered Report received an in-principle acceptance, on DATE. Data collection took place during a one year window initiating on DATE and ending on DATE. Prior to receiving an in-principle acceptance, we made an announcement on relevant listservs (i.e., cogdevsoc and infancy) to gauge general interest in the project, as having a sense of the number of labs that might be interested in participating helped us plan methodological decision making (i.e., whether we would have sufficient power to propose two experimental conditions and whether labs would be using one versus two experimenters). At the time we submitted the Stage 1 version of this Registered Report, sixty-one labs in seventeen countries expressed interest in collecting data, pledging to test a total of 1,414 infants.

Age distribution. Participating labs were asked to recruit participants between 5.5 months (167 days) and 10.5 months (319 days), covering a 5-month age window.

Power. Findings from the meta-analysis by Margoni and Surian (2018) revealed that the mean proportion of infants' preference for the Helper was $0.64,95 \%$ CI $[0.60,0.69]$. Using the Bayesian analysis tools described below, we computed the number of participants necessary to have a .8 probability (corresponding to $80 \%$ power) to find evidence against the null hypothesis of no preference between Helpers and Hinderers, indicated by a Bayes factor greater than 3 (Kruschke \& Liddell, 2018). In our simulations, this probability was achieved by including 140 participants in a study with a single condition (i.e., the Social condition). To achieve the same probability in a study designed to detect a difference between an experimental group that shows 


\section{INFANTS' SOCIAL EVALUATIONS}

an effect and a control group that performs at chance (i.e., the Non-Social control condition), our simulations revealed that 500 participants were needed.

Lab participation criteria. Since the precision of our analysis is affected by the number of labs contributing data more than by the number of participants within each lab (Judd, Westfall, \& Kenny, 2017), and since including a large number of labs is important to the longterm objective of building a diverse community of researchers to engage in replication projects, we adopted a liberal inclusion criterion. We specified that labs should recruit typicallydeveloping infants within the specified age range (see below for full list of demographics collected). Sample sizes were asked to be calculated on the basis of the total number of infants who saw the entire video presentation, made a choice, and did not fit any of the exclusion criteria (described in detail below), meaning that most labs would likely need to recruit more than 16 infants. Labs were required to make sample size decisions prior to testing. If labs were unable to achieve their initial goal of testing at least 16 infants, or if they tested more participants than they had initially registered for, we included their data as long as their decision to test those participants was not based on results (e.g., whether or not an expected effect was observed).

Ethics. Prior to collecting data, all labs were required to agree to a "Code of Conduct" where they agree to maintaining a high level of integrity and ethics in their involvement in the project, including the explicit, detailed information about data collection integrity in the project instruction manual (e.g., not making decisions about stopping/extending data collection based on the data itself). Labs acquired their own Ethics Review Board protocols for data collection. All central data analyses used de-identified data. Individual video recordings of participants were coded and stored at each individual lab. In addition, if permitted by individual laboratories' Ethics protocols, participant videos were uploaded to a centralized video library accessible by 


\section{INFANTS' SOCIAL EVALUATIONS}

other co-investigators called DataBrary (https://nyu.databrary.org/), a video data library used by behavioral scientists world-wide. Since not all laboratories had permission to share their videos, all primary data coding and exclusion criteria were determined by individual laboratories.

Lab Research Practices. Labs were instructed to follow their individual protocols for training of research assistants and to maintain the same quality standards for this study as for other studies they conduct. Each lab completed a general questionnaire to report on their training practices, academic standing and experience of the experimenters involved in the present study (e.g., volunteer, undergraduate, graduate, post-doctoral, professor), and protocol for greeting families. Additionally, laboratories were required to create "lab tour videos" in which they walked through their lab setup and experimental procedure. These videos were uploaded to a central database.

Lab Training and Reliability. Prior to initiating data collection, labs were required to send three videos of the interactive part of the procedure (i.e. the "choice phase") to a centralized team of researchers for approval. To be approved, the pilot videos were required to follow the procedures outlined in an Experimenter's Manual (e.g., timing of verbal prompts, presentation distance and angle of choice characters, see Appendix A for full instructions). This review procedure was implemented to ensure labs strictly adhered to the experimental protocol. The decision to switch from piloting to data collection within each lab was made prior to data collection, and no pilot data is included in the final analyses.

To ensure standardization of coding for the primary variable of interest (i.e., infants' choice of puppet during the test phase), we created a centralized training process for choice coding using a centralized bank of videos with varying levels of coding difficulty. Prior to collecting data, all labs were required to complete this training with a reliability of $90 \%$. The full 


\section{INFANTS' SOCIAL EVALUATIONS}

protocol can be found in [will be uploaded with final submission]. During data collection, the experimenter recorded and coded the infants' choice behavior. Offline, a second researcher naive to condition coded infants' choices from video as a reliability check for the experimenter's online coding. If the offline coder disagreed with the experimenter, the offline coder's choice was used for the analyses. Labs were required to report the percent agreement between the two coders in their final data reporting.

Labs were not required to participate in a standardized training process for coding looking-time data because some labs used automated techniques for coding (e.g., eye-tracking) and looking-time was not the primary variable of interest in this experiment. Regardless, we asked all labs to conduct their own reliability coding for looking-time data within their lab on $25 \%$ of their sample and upload this data to the project's data repository.

\section{Participants}

A total of XYZ typically developing, full-term infants participated from XYZ labs (mean laboratory sample size $=X Y Z, S D=X Y Z$, range: $X Y Z)$. Information on all participating and included labs is provided in Table 1 . The average age of infants included in the study was XYZ days (SD: XYZ, range: XYZ), and XX\% were female. XYZ additional infants were tested but not included in the final sample due to their having met exclusion criteria (see below).

\section{Procedure}

Procedural instructions provided to each participating lab are viewable in the Supplemental Materials Appendix F. Infants were seated in front of the screen, either in an infant seat or on their parent's lap. They were randomly assigned to one of two conditions, a Social condition or a Non-Social control condition (described below). The conditions differed only in the content of the video stimuli - the overall procedure was otherwise identical across conditions. 


\section{INFANTS' SOCIAL EVALUATIONS}

While the video stimuli were displayed, parents were asked to close their eyes or wear occluding glasses. Parents were also given a set of standardized instructions on how to maintain proper positioning throughout the experiment so that infants could fully view the display and to avoid inadvertently biasing their infant: they were told to sit still (e.g., act like infants' "chairs", try to keep them in their lap, and hold them around the rib cage), not talk or gesture during the experiment, and not redirect infants' attention to the events if the infants grew uninterested. Infants were video recorded during the entire experiment.

Apparatus. Laboratories were instructed to display the video stimuli using the setup with which they were most familiar (e.g., TV screen, projection screen, computer monitor, etc.). The

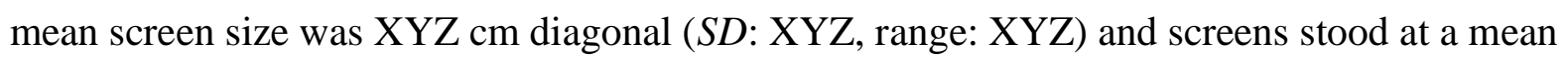
distance of XX cm (SD: XYZ, range: XYZ) from infants. Laboratories were instructed to position infants' faces at the center of the screen so they were not required to look up or down to view the stimuli. To facilitate standardization across labs and experimenter blinding, laboratories were encouraged to use PyHab (Kominsky, 2019) to display the video stimuli. PyHab is a free software program based in a PsychoPy environment (Kanbe, 2019; Peirce, 2019) that randomly selects a counterbalanced order, presents stimuli, and records looking time, all while allowing the experimenter to remain blind to the onscreen stimuli display. Through this procedure, the experimenter is able to watch the infant's face, initiate a trial by pressing a key, register when the infant is looking at the screen by the pressing and release of a key. Pyhab expects the experimenter and the infant to be viewing separate monitors, and rather than viewing what the infant is watching, the experimenter sees a display that simply indicates whether a trial is active or not. In this set up, it is possible for the entire experiment to be run by a single experimenter who is unaware of the puppets' identities (i.e. which puppet is the Helper vs. Hinderer). Labs that 


\section{INFANTS' SOCIAL EVALUATIONS}

did not use PyHab were instructed to reproduce these testing conditions as closely as possible, and were strongly encouraged to use a second experimenter whenever possible in order to ensure the experimenter remained naive to the puppets' identities. Regardless of set up, we required that the experimenter administering the choice phase be naive to puppet identity. Thus, if labs did not use PyHab or a second experimenter, they were required to report how they ensured the experimenter remained naive to the puppets' identities.

Personnel. Labs were given the option of running the experiment with one or two experimenters, as long as the experimenter administering the choice phase was blind to the puppets' helping/hindering identities. [insert breakdown of experimenter status here. e.g., undergraduate student, graduate student, PI].

Calibration. Prior to displaying the habituation trials, an attention-getting stimulus (a spinning multi-colored circle) appeared in turn on each of the four corners of the screen and once in the center, allowing the online coder to calibrate infants' looking to the screen. The attentiongetter moved to a new location after the experimenter pressed a key indicating that the infant looked at it.

Onset of each trial. Each trial began with an attention-getting stimulus at the center of the screen (e.g. a twirling/squeaking multi-colored circle). When the experimenter determined that the infant fixated on the screen, the experiment proceeded to the next trial.

Habituation phase. The stimuli (Figure 2) used for habituation were modeled off those in Hamlin and colleagues (2007), and are viewable at the following link in the folder "Video Files March 2021"(https://osf.io/xe2pj/files/). The habituation phase of the experiment was the only aspect of the study that differed between the Social and Non-Social conditions. 


\section{INFANTS’ SOCIAL EVALUATIONS}

Habituation Phase: Social Condition. Stimuli consisted of four 16-second, pre-recorded, live-action puppet shows displayed via video depicting either a "helping event" or a "hindering event". Each event was filmed against a white background and featured a green hill rising from the bottom right to the top left of the scene. The hill had two inclines, the second steeper than the first, with a small flat plateau between them. Characters consisted of three colored wooden shapes (i.e., a red circle, a blue square, and a yellow triangle), each featuring googly eyes. The red circle was always the "Climber" character who tried but failed to climb the hill; the Climber's eyes were fixed pointing upward so that it continuously gazed toward her goal. The blue square and yellow triangle were the "Helper" and "Hinderer" characters; their eyes were not fixed and the characters' identities were counterbalanced across participants. Soft instrumental music played in the background of the events to maintain infants' interest.

For each event, the Climber was observed trying but failing to reach the top of the hill. The Climber was first shown resting at the bottom of the hill, at the right edge of the display. The Climber then moved up the first mild incline to a plateau, where it "danced" briefly. Next, the Climber attempted but failed twice to reach the top of the second, steeper incline, moving slightly higher but sliding back down to the plateau after each attempt. During the Climber's third attempt, a second agent (the Helper or the Hinderer) entered the scene. In order to draw infants' attention to the screen just before the critical "hitting" aspects of the events occured, a “ding” sound was played 300 ms prior to the second agent's appearance on stage. During helping events, the Helper entered the scene from the bottom of the hill, moved up the hill, and eventually contacted the Climber. The Helper hit the Climber twice from below, pushing it upward to the top of the hill. During hindering events, the Hinderer entered the scene from the top of the hill and moved down, eventually contacting the Climber. The Hinderer hit the Climber 


\section{INFANTS' SOCIAL EVALUATIONS}

twice from above, pushing it downward to the bottom of the hill. Once the climber reached its final destination (the top or bottom of the hill), the Helper or Hinderer exited the stage from where they entered and the event paused. This last frame remained on the screen until either the infant looked away for 2 consecutive seconds or 30 seconds elapsed (Hamlin, 2015). The helping and hindering events were matched in timing, speed, and sound down to the millisecond (see above OSF link for a comparison of videos). For example, the timing of the initial attempts of the Climber, the first frame where the Helper/Hinderer enters the stage, the first contact between the Helper/Hinderer and the Climber, and the exit speed of the Helper/Hinderer are identical across videos. All variations of the videos used the same audio track.

To ensure that infants viewed the helping/hindering events in their entirety, we identified a Critical Period from the first frame the Helper or Hinderer appeared on stage to the last frame where the Helper or Hinderer was moving (2,500 ms). If infants looked away during the Critical Period for more than 1,000 cumulative ms (i.e., $40 \%$ of the window), the trial was terminated and repeated up to three times ${ }^{1}$. Failure to attend to the events within this Critical Period after three showings resulted in the exclusion of the infant due to inattentiveness. If such occurred within the first six trials, the experiment was nevertheless continued until the 6th trial and ended immediately after. This was done to avoid any frustration for caregivers that could arise from ending the experiment abruptly, and to ensure that each family had the opportunity to fully participate in the experiment.

Helping and hindering trials were shown in alternation until infants fulfilled the preset habituation criteria, adopted from Hamlin and colleagues (2007). To fulfill these criteria, infants' summed looking times to any three consecutive trials had to decrease to less than half the

\footnotetext{
${ }^{1}$ Pilot testing revealed that brief look-aways outside this Critical Period were common, especially during trials near the end of the habituation phase.
} 


\section{INFANTS' SOCIAL EVALUATIONS}

summed looking time to the first three trials for which looking totaled 12 seconds or more. Once this criterion was met, or after a maximum of 14 trials, infants moved on to the Choice Phase. The presentation order of the videos (helping first versus hindering first) was counterbalanced across participants.

Habituation Phase: Non-Social Control Condition. Infants viewed events highly similar to the helping and hindering events of the Social condition but with one critical difference: the Climber was replaced by an inanimate, eyeless object that did not exhibit self-propelled motion. Because there were no initial climbing actions, videos in the Non-Social condition were $4.4 \mathrm{~s}$ shorter in duration than those in the Social condition. In these videos, infants viewed an inert red ball get pushed up or down the hill by the same animate triangle and square characters from the Social condition. Aside from these differences, the videos across conditions were closely matched on visual and sound cues. The screen time of the square/triangle characters was closely matched across conditions (i.e., screen time of helper/hinderer: $4.7 \mathrm{~s}$ per video; screentime of up-pusher/down-pusher: $5.9 \mathrm{~s}$ per video). The critical window was defined using the same boundaries as the Social condition: it started from the first frame the Helper or Hinderer appeared on stage to the last frame when the Helper or Hinderer stopped moving $(3.5 \mathrm{~s})$. As in the Social condition, if infants looked away for more than $40 \%$ cumulative ms during this critical window (i.e., 1,400 ms), the trial was terminated and repeated up to three times. The habituation criteria were identical across conditions. Within the Non-Social condition, pushing up versus down videos were closely matched on speed, timing, and sound cues. The presentation order of the videos (pushing up versus down first) was counterbalanced across participants.

Choice Phase. Complete instructions for administering the choice phase are viewable in Appendix A of the Supplemental Materials. The Choice Phase was identical across conditions. 


\section{INFANTS' SOCIAL EVALUATIONS}

Immediately following the end of the habituation phase, an experimenter (naive to the identity of the characters) presented infants with foam versions of the yellow Triangle and blue Square characters, attached with Velcro to a $45 \mathrm{~cm}$ x $60 \mathrm{~cm}$ board with a white background (Figure 1). The characters were standardized in size: blue square, $9.9 \mathrm{~cm} \mathrm{x} 9.9 \mathrm{~cm}$, and yellow triangle, $14.8 \mathrm{~cm}$ (base) x $13 \mathrm{~cm}$ (height). Labs were provided with hyperlinks to the material used and templates for creating the characters (see Appendix B for excerpts from the links). A subset $(\mathrm{XX} \%)$ of characters were centrally created by the project team and distributed to some participating labs via mail or at the International Congress for Infancy Studies conference in July 2018. Each character was located $7.5 \mathrm{~cm}$ from the base to the bottom of the board. Velcro pieces were placed at the center of each character. Measuring from the center of the Velcro, the pieces were $30 \mathrm{~cm}$ apart from each other, $15 \mathrm{~cm}$ from each side of the board, with each character placed $\sim 9 \mathrm{~cm}$ from its outermost point to the side edges of the board. The location of characters (left/right) was counterbalanced across participants.

At the start of the Choice Phase, parents were instructed to grasp their infants securely around the waist and position them close to their knees to facilitate infants' reaching. Parents were then asked to close their eyes or wear occluding glasses to prevent them from biasing the child's attention towards a particular character.

Next, the experimenter leaned over in front of the infant and said, "Hi! Look!," while lowering the board directly at an approximately 30-degree angle. The board was placed just out of the infant's reach until the infant looked at both characters. When the infant had done so, the experimenter said, "Hi!" to direct the infant's attention away from the characters and back to the experimenter. Upon making eye contact with the infant, the experimenter said, "Who do you like?" while moving the board within the infant's reach. The experimenter kept the board 


\section{INFANTS' SOCIAL EVALUATIONS}

extended towards the child for $60 \mathrm{~s}$, while keeping track of time either in their head, using a stopwatch, or by referencing a wall clock. Any choices that were made after $60 \mathrm{~s}$ were excluded from the analyses (e.g., in the case the experimenter inadvertently extended the choice phase past $60 \mathrm{~s})$.

Infants' choice of character was coded online by the experimenter conducting the Choice Phase. A visually guided reach (touching one character while looking at it) was indicative of infants' choices. Occasionally, infants touched both puppets at once; some of these instances were counted as valid choices according to our predefined criteria. In usable both touches, the infant clearly directed her gaze and reached towards one character, but touched the other character as well. On the other hand, unusable both touches involved the infant touching both characters with unclear or inconsistent visual attention. Experimenters were instructed to continue the choice procedure until a usable touch was recorded by encouraging and reprompting the baby after $30 \mathrm{~s}$ had passed. If infants did not make a choice during the $60 \mathrm{~s}$ Choice Phase, their data were excluded from final analyses. Experimenters were required to administer the choice phase within two minutes of ending the habituation videos. If more than two minutes passed the session was considered an "experimenter error" and excluded from data analysis.

Order of testing. Laboratories occasionally tested participants in a separate (unrelated) experiment during their visit. We encouraged, but did not require, labs to run the Helper/Hinderer experiment as their first experiment. All labs recorded whether another experiment was run with the same participants and whether it preceded or followed the Helper/Hinderer experiment.

Demographics. Each lab collected a set of participant demographic information for each infant: gender, date of birth, estimated proportion of language exposure for language(s) heard 


\section{INFANTS' SOCIAL EVALUATIONS}

daily, preterm/full-term status (i.e., more than 36 weeks gestation), hearing or visual impairments, developmental concerns (e.g., developmental disorders), infant handedness (right, left, not sure), parent handedness (right, left), and color blindness in the immediate family. Labs were given a standard participant questionnaire that they were encouraged to use (see Appendix C of the Supplemental Materials).

\section{Exclusion Criteria}

All data collected for the study (i.e., every infant for whom a data file was generated, regardless of how many trials were completed) were uploaded to the central database for analysis. We instructed labs to make note of any instances in which a procedural error or anomaly occurred during testing. Participants were excluded from the final analysis if they met any of the criteria below.

1. Not full-term. Full term was defined as 36 weeks or more gestation. Caregivers were asked to report their child's due date and a centralized research team calculated the child's gestational age. XYZ (XYZ\%) of tested infants were excluded for this criterion.

2. Diagnosed developmental disorders. If parents reported any known developmental disorders their infants were excluded. XYZ (XYZ\%) of tested infants were excluded for this criterion.

3. Failure to set a habituation criterion. Infants set the habituation criterion if their looking time towards the paused frame at the end of the habituation video on any 3 consecutive trials during the first 6 trials summed to at least 12 seconds. XYZ (XYZ\%) of tested infants were excluded for not meeting this criterion.

4. Failure to view the Critical Period. If infants looked away for more than $750 \mathrm{~ms}$ cumulatively during the Critical Period of a trial, the experimenter repeated the trial up to 


\section{INFANTS’ SOCIAL EVALUATIONS}

two times. If infants failed to attend to aCritical Period after three consecutive trials, they were excluded. XYZ (XYZ\%) of tested infants were excluded for this criterion.

5. Failure to make a clear choice. Infants must have produced visually guided reaches (as defined in the Supplementary Materials, Appendix A) towards one of the two characters in the Choice Phase. XYZ (XYZ\%) of tested infants were excluded for this criterion.

6. Parental/outside interference. Infants were excluded if distracting events that were not part of the study protocol occurred during the Habituation or Choice Phase (e.g., a noise outside the testing room, the parent gesturing to the child). XYZ (XYZ\%) of tested infants were excluded for this criterion.

7. Experimenter error. Experimenter errors included any actions the experimenter inadvertently made that may have influenced infants' behavior (e.g., the experimenter failed to record an infant's looking time, or became aware of puppets' identity prior to the Choice Phase). XYZ (XYZ\%) of tested infants were excluded for this criterion.

8. Equipment error. Any technical deviations that may have influenced infants' behavior were considered equipment errors (e.g., stimulus froze during presentation). XYZ (XYZ\%) of tested infants were excluded for this criterion.

\section{Results}

Note that some analyses may appear in online supplemental materials rather than in the main text.

Note: Pilot data only include infants in the Social condition, as the decision to run a Non-Social control condition was made during the peer review process, after initial piloting was complete. These pilot data will therefore be reported in Supplementary Materials only (see Appendix E). Data simulations with the expected effect size were used to confirm the validity of our analysis 
INFANTS' SOCIAL EVALUATIONS

structure to test the effects of interest. All analysis scripts, including simulations for Bayesian power analyses and choices for the statistical plan, can be found online (https://github.com/manybabies/mb4-analysis). Bayesian power analysis was conducted in a similar fashion to frequentist power analysis, with the difference that power was estimated as the number of samples in which Bayes factors were greater than the threshold value 3.

\section{Analysis structure}

We used a Bayesian analysis framework to calculate Bayes factors through Bayesian model comparisons. This framework provides a principled method for testing evidence in favour of and against the null hypothesis (i.e., no difference in preference for helpers over hinderers), and allowed us to fit hierarchical models to account for sparse data and variation across labs.

We fit generalised linear mixed-effects Bayesian models using Stan (Gelman, Lee, \& Guo, 2015) and the brms package (version XXX; Bürkner, 2017); 95\% Credible Intervals (CI) were computed using the HPDInterval function from the coda package (version XXX; Plummer, Best, Cowles, \& Vines, 2006). While standard frequentist 95\% Confidence Intervals are defined such that if repeated samples are drawn from a population, the Confidence Interval will include the true population mean in $95 \%$ of the observed samples, Bayesian Credible Intervals represent the interval in which the true population mean is likely to be, given the data at hand and the model assumptions (Morey et al., 2016).

We were also interested in assessing the evidence for specific hypotheses (e.g., a nonzero preference for helpers over hinders). While Bayesian CIs allow us to assess the precision of our measurement of effects, they do not allow for assessment of evidence in this way. For this purpose, Bayes factors (BFs) are the appropriate Bayesian tool. Crucially, while $p$-values only allow us to reject the null hypothesis H0, BFs obtained through model comparison allow us to 


\section{INFANTS' SOCIAL EVALUATIONS}

either reject $\mathrm{H} 0$ in favor of $\mathrm{H} 1$, accept $\mathrm{H} 0$ to the detriment of $\mathrm{H} 1$, or conclude that the data do not provide sufficient evidence to support either. To obtain BFs, we computed two models for each specific research question, one representing the null hypothesis $\left(\mathrm{H}_{0}\right)$ and the other an alternative hypothesis $\left(\mathrm{H}_{1}\right)$. We then estimated the posterior distributions of each model and compared them to obtain a BF, using the bridgesampling package (version XXX; Gronau, Singmann, \& Wagenmakers, 2017).

\section{Confirmatory analyses}

Our primary research questions were (1) whether there was an above-chance choice of the Helper in the Social condition compared to the Non-Social control group, and (2) whether there were developmental differences in choice. Based on the original study (Hamlin et al., 2007), we predicted that the proportion of children choosing the Helper would be above chance in the Social condition, and that age would not moderate the effect size.

Note: Depending on the total amount of data collected, we propose two analysis plans with power analyses computed for each plan (reported below). In the event that we do not have enough participants to reach $80 \%$ power to compare the Social to the Non-Social condition $\left(n_{\min }\right.$ $=500)$, we will revert to testing the Social condition only against chance $\left(n_{\min }=140\right)$. Below, we describe the analysis plan with a Non-Social control group. In case our sample size is only sufficient for comparing the choices of infants in the social group against chance performance, the same procedure will be used, removing the main effect of condition from the model, and instead testing for the intercept.

We tested our prediction via a Bayesian generalized linear mixed effects model with a Bernoulli response model. In such models, the probability for the dependent variable (here, participants' choice) is transformed through the logit function such that an estimate of zero 


\section{INFANTS' SOCIAL EVALUATIONS}

corresponds to a $50 \%$ chance for either choice, with values greater than zero representing a preference for the Helper. The specification of our model was:

$$
\begin{aligned}
& \text { choice } \sim 1+\text { condition + age + condition:age + } \\
& (1+\text { condition + age + condition:age / lab })
\end{aligned}
$$

Choice (Helper versus Hinderer) and condition (social versus non-social) were entered as binary variables. Age (in days) was scaled and centred to allow for better convergence of the statistical models and easier interpretation of the results. This type of variable scaling is standard (e.g., Marquart, 1980) and we adopt it because (a) keeping raw age in days would result in a meaningless intercept and main effect of condition (representing a hypothetical preference for either group at age $=0$ ), and (b) estimating an intercept and a difference between conditions at age $=0$ as well as a slope for age would lead to less precise estimates (as we found in simulations). To control for possible variation in preference across labs as well as in developmental trends across labs, the model also included random intercepts for each lab and random slopes for condition, age, and their interaction, by lab.

In Bayesian analyses, the most appropriate method for pooling data from a new experiment with previous knowledge is to set priors based on that previous knowledge. For our key effect of interest, namely the effect of condition (a higher or lower proportion of Helper choices in the Social versus Non-Social group), we used an informative normal prior based on the effect size and confidence interval from the meta-analysis of Margoni and Surian (2018). We used the following formula to compute the standard deviation from the reported confidence interval $\left(\sigma=C I_{\text {one-side width }} \times \sqrt{N_{\text {mean }} / z(95)}\right.$; here, mean $=\operatorname{logit}(0.64)=0.58$, standard deviation $=0.1$. We believe that this choice was warranted given that the meta-analysis included both studies which showed and did not show the hypothesized effect, as well as published and 


\section{INFANTS' SOCIAL EVALUATIONS}

unpublished data, making them unlikely to reflect the outcome of a biased literature selection towards the effect of interest. Further, the estimate of .64 represents the proportion of infants choosing prosocial characters after having corrected for possible publication bias. Sensitivity analyses using non-informative priors are presented alongside our results. For these analyses, we only specify a prior on the random effects to improve model convergence.

We further used weakly informative normal priors for the intercept (mean $=0$, standard deviation $=0.1)$, relationship of age to choice and its interaction by condition $($ mean $=0$, standard deviation $=.5$ ), and a restricted Student prior on random effects (as compared to the default in the brms package). Priors of this type provide very little information in cases like ours where we have large amounts of data; their primary purpose is to improve model convergence and subsequent bridge sampling for the computation of BFs.

Relative to the first research question, the main result we aimed to reproduce here was infants' preference for the Helper at levels greater than chance, as reflected by a greater than zero estimate for the effect of condition. To assess the evidence for this hypothesis, we computed the $\mathrm{BF}$ in favor of the full model described above $\left(H_{1}\right)$ compared to a model that did not include a main effect of condition or any higher level terms including this effect $\left(H_{0}\right)$. Notably, random effects corresponding to the dropped fixed effects were also dropped. ${ }^{2}$ Indeed, random effects only make sense with respect to the corresponding fixed effects, and thus should not be kept for nested model comparisons. More precisely, the model for $H_{0}$ was specified as:

$$
\text { choice } 1+\text { age }+(1+\text { age } \mid \text { lab })
$$

\footnotetext{
${ }^{2}$ We are currently running simulations to determine whether it is more appropriate to keep or drop random effects from our model comparisons. We will choose the model comparison method that yields the best Type I and II error rates, so long as these models successfully converge.
} 


\section{INFANTS’ SOCIAL EVALUATIONS}

Following Kass and Raftery (1995), we interpreted a BF > 3 in favor of either $H_{0}$ or $H_{1}$ as substantial evidence and $\mathrm{BF}>10$ as strong evidence ${ }^{3}$. We found a $B F_{10}=234.61$ (140.67 with non-informative priors) in favor of $H_{1}$, suggesting that the addition of a main effect of condition, as well as the condition-by-age interaction, increased model fit. In other words, infants preferentially chose the Helper versus Hinderer agent, which replicates previous findings. [As a reminder, these results are obtained from data simulated according to the previous findings, it is thus not surprising that we find replicated results. With real data, in the unlikely case of a BF < 3 and $>.33$, we will write: These results are qualified by the low overall level of evidence on this question provided by our data.] See Figures 3 and 4. Parameter estimates for this model with and without informative priors are reported in Table 2 and Table 3, along with estimated error and 95\% Credible Intervals (CI) for those parameters.

Relative to the second research question, we were interested in whether the addition of the condition-by-age interaction to the model contributed to model fit. We assessed evidence for this question via the same procedure as above, fitting a null model without age:

choice $\sim 1$ + condition + age + (1 + condition + age | lab)

The Bayes Factor for this comparison was $\mathrm{BF}_{10}=0.019$ (0.023 with non-informative priors), suggesting that the condition-by-age interaction did not contribute to model fit. In other words, the preference for the Helper in the social group was not mediated by age. [We will interpret the direction and magnitude of the age coefficient if $|B F|>3]$.

As a follow-up analysis, we refitted the same model including only infants who successfully habituated to the events. We again fitted a Bayesian Bernoulli linear mixed effects model. Choice (Helper versus Hinderer) and condition (Social versus Non-Social) were entered

\footnotetext{
${ }^{3}$ The notation $B F_{10}$ refers to the Bayes Factor for the alternative hypothesis. Conversely, the notation $B F_{01}$ refers to the Bayes Factor for the null hypothesis.
} 


\section{INFANTS' SOCIAL EVALUATIONS}

as binary variables. Age (in days) was scaled and centered. To control for possible variation in preference across labs as well as in developmental trends across labs, the model also included random intercepts for each lab and random slopes for condition and age by lab. Priors were as above. We used the same model comparisons and the same method to obtain BFs. Note: this sub-analysis cannot be satisfactorily run using our simulated data, thus we do not give an example report of the result. It will follow the same structure as for the main analysis described above.

Finally, we were interested in variation in preference scores across labs. Following Klein et al. (2014), we calculated the binary intraclass correlation coefficient using the ICCbin package in R (version XXX; Chakraborty \& Hossain, 2018). This measure captures the degree to which the proportion of Helper choices was correlated across labs. [REPORT AND INTERPRET ICC].

\section{Exploratory Analyses}

An additional set of exploratory analyses may be run as well. Example analyses that may potentially be run with the dataset include: the relation between choice behavior and (1) attention to the video events (e.g. as measured by the number of trials to habituation, overall looking time to the still frame events), (2) clear versus ambiguous choice actions, and (3) experimenter blindness (i.e., whether the experimenter administering the choice phase knew whether the infant participated in the Social vs. Non-Social condition). To test for the possibility of attentional differences across the Social and Non-Social conditions, we plan to conduct exploratory analyses of infants' gaze behavior during habituation, and in particular how long they look at the still image of the final frame presented after each video. If attentional differences are found, we intend to further assess whether these differences predict choice behaviour, to control for the 


\section{INFANTS' SOCIAL EVALUATIONS}

possibility that infants' different performance in the Social and Non-Social condition may be due to attentional differences during habituation.

\section{Topics to Include in Discussion}

- Summary of key findings as they relate to the central questions outlined in the introduction.

- Two key points to discuss: (1) How replicable are the findings and what factors moderate the effect and (2) if the findings are replicable, why previous replication attempts might have failed (and may fail again), and what the findings might mean (rich vs. lean interpretations): Minimally, these behaviors show that infants can discriminate helping/hindering actions, distinguish them from perceptually similar nonsocial actions, and selectively make a choice based on this. At a higher-level, these behaviors may support the hypothesis that socio-moral cognition, similar to other conceptual domains (e.g., objects, number), is a core aspect of human cognition (Hamlin, 2013; Kinzler \& Spelke, 2007). Because the current research is unable to speak to these broader theoretical issues, additional research in this area is needed.

- Discuss the importance of replication in science. Replication is key to scientific progress in that it (1) establishes the accuracy of a finding, (2) examines the conditions under which the finding is observed, and (3) approximates the finding's true, underlying effect size (Open Science Collaboration, 2015; Zwaan et al., 2018).

\section{Note on Additional Questions of Interest}

There are many research questions that might be pursued in follow-up, "spin-off", projects that fall outside the scope of the current paper. These projects will require additional coding of the collected data (by a centralized group of researchers). Example projects might 


\section{INFANTS' SOCIAL EVALUATIONS}

explore how infants' choice during test relates to: infants' behavior during the choice phase (e.g. latency to make a choice, touching both characters during test), infants' affect while watching the videos, and patterns of gaze fixation during video presentations (for labs using eye tracking). They will not be addressed here. 
INFANTS’ SOCIAL EVALUATIONS

\section{References}

Abramson, L., Dar, M., Te’eni, A., \& Knafo-Noam, A. (2016). Preferences for helpers and hinders in 9- and 18-month-old infants. Unpublished raw data.

Brandone, A. C., \& Wellman, H. M. (2009). You can't always get what you want: Infants understand failed goal-directed actions. Psychological science, 20(1), 85-91.

Brownell, C. A. (2013). Early development of prosocial behavior: Current perspectives. Infancy, 18(1), 1-9. doi: 10.1111/infa.12004

Buon, M., Jacob, P., Margules, S., Brunet, I., Dutat, M., Cabrol, D., \& Dupoux, E. (2014). Friend or foe? Early social evaluation of human interactions. PloS One, 9(2), e88612.

Bürkner, P.-C. (2017). brms: An R Package for Bayesian Multilevel Models Using Stan. Journal of Statistical Software, Vol. 80. https://doi.org/10.18637/jss.v080.i01

Burns, M. P., \& Sommerville, J. A. (2014). "I pick you": the impact of fairness and race on infants' selection of social partners. Frontiers in Psychology, 5, 93.

Byers-Heinlein, K., Bergmann, C., Davies, C., Frank, M. C., Hamlin, J. K., Kline, M., Kominsky, J. F., Kosie, J. E., Lew-Williams, C., Liu, L., Mastroberardino, M., Singh, L., Waddell, C. P. G., Zettersten, M., \& Soderstrom, M. (2020). Building a collaborative psychological science: Lessons learned from ManyBabies 1. Canadian Psychology/Psychologie Canadienne, 1-51. doi: 10.1037/cap0000216

Carpendale, J. I., \& Lewis, C. (2004). Constructing an understanding of mind: The development of children's social understanding within social interaction. Behavioral and brain sciences, 27(1), 79-96. 


\section{INFANTS' SOCIAL EVALUATIONS}

Carter, E. C., Schönbrodt, F. D., Gervais, W. M., \& Hilgard, J. (2019). Correcting for bias in psychology: A comparison of meta-analytic methods. Advances in Methods and Practices in Psychological Science, 2(2), 115-144. doi: 10.1177/2515245919847196

Chae, J. J. and Song, H. (2018), Negativity bias in infants' expectations about agents' dispositions. British Journal of Developmental Psychology, 36, 620-633. doi:10.1111/bjdp. 12246

Chakraborty, H., \& Hossain, A. (2018). R package to estimate intracluster correlation coefficient with confidence interval for binary data. Computer Methods and Programs in Biomedicine, 155, 85-92.

Dahl, A., Schuck, R. K., \& Campos, J. J. (2013). Do young toddlers act on their social preferences? Developmental Psychology, 49(10), 1964-1970.

Duval, S., \& Tweedie, R. (2000). A nonparametric "trim and fill" method of accounting for publication bias in meta-analysis. Journal of the American Statistical Association, 95:449, 89-98. doi: 10.1080/01621459.2000.10473905

Fehr, E., \& Fischbacher, U. (2003). The nature of human altruism. Nature, 425(6960), 785-791.

Frank, M. C., Bergelson, E., Bergmann, C., Cristia, A., Floccia, C., Gervain, J., .. Yurovsky, D. (2017). A collaborative approach to infant research: Promoting reproducibility, best practices, and theory-building. Infancy, Vol. 22, pp. 421-435. doi: 10.1111/infa.12182

Freud, S. (1961). The ego and the id. In The Standard Edition of the Complete Psychological Works of Sigmund Freud, Volume XIX (1923-1925): The Ego and the Id and Other Works (pp. 1-66). 


\section{INFANTS' SOCIAL EVALUATIONS}

Gelman, A., Lee, D., \& Guo, J. (2015). Stan. Journal of Educational and Behavioral Statistics: A Quarterly Publication Sponsored by the American Educational Research Association and the American Statistical Association, 40(5), 530-543.

Geraci, A., \& Surian, L. (2011). The developmental roots of fairness: infants' reactions to equal and unequal distributions of resources. Developmental Science, 14(5), 1012-1020.

Gronau, Q. F., Singmann, H., \& Wagenmakers, E.-J. (2017). bridgesampling: An R Package for Estimating Normalizing Constants. doi: 10.31222/osf.io/v94h6

Hamlin, J. K. (2015). The case for social evaluation in preverbal infants: gazing toward one's goal drives infants' preferences for Helpers over Hinderers in the hill paradigm. Frontiers, 5, 1563. doi: 10.3389/fpsyg.2014.01563

Hamlin, J. K. (2013). Failed attempts to help and harm: intention versus outcome in preverbal infants' social evaluations. Cognition, 128(3), 451-474.

Hamlin, J. K., Hallinan, E. V., \& Woodward, A. L. (2008). Do as I do: 7-month-old infants selectively reproduce others' goals. Developmental science, 11(4), 487-494.

Hamlin, J. K., Ullman, T., Tenenbaum, J., Goodman, N., \& Baker, C. (2013). The mentalistic basis of core social cognition: experiments in preverbal infants and a computational model. Developmental Science, Vol. 16, pp. 209-226. https://doi.org/10.1111/desc.12017

Hamlin, J. K., \& Wynn, K. (2011). Young infants prefer prosocial to antisocial others. Cognitive Development, 26(1), 30-39. doi: 10.1016/j.cogdev.2010.09.001

Hamlin, J. K., Wynn, K., \& Bloom, P. (2007). Social evaluation by preverbal infants. Nature, 450(7169), 557-559. 


\section{INFANTS' SOCIAL EVALUATIONS}

Hamlin, J. K., Wynn, K., \& Bloom, P. (2010). Three-month-olds show a negativity bias in their social evaluations. Developmental Science, 13(6), 923-929.

Hamlin, J. K., Wynn, K., Bloom, P., \& Mahajan, N. (2011). How infants and toddlers react to antisocial others. Proceedings of the National Academy of Sciences of the United States of America, 108(50), 19931-19936.

Holvoet, C., Scola, C., Arciszewski, T., \& Picard, D. (2016). Infants’ preference for prosocial behaviors: A literature review. Infant Behavior \& Development, 45(Pt B), 125-139.

Judd, C. M., Westfall, J., \& Kenny, D. A. (2017). Experiments with More Than One Random Factor: Designs, Analytic Models, and Statistical Power. Annual Review of Psychology, $68,601-625$.

Kanakogi, Y., Inoue, Y., Matsuda, G., Butler, D., Hiraki, K., \& Myowa-Yamakoshi, M. (2017). Preverbal infants affirm third-party interventions that protect victims from aggressors. Nature Human Behaviour, Vol. 1. https://doi.org/10.1038/s41562-016-0037

Kanbe, F. (2019). Generating Strictly Controlled Stimuli for Figure Recognition Experiments. Journal of Visualized Experiments. doi:10.3791/59149

Kass, R. E., \& Raftery, A. E. (1995). Bayes factors. Journal of the American Statistical Association, 90(430), 773-795. doi:10.2307/2291091

Kinzler, K. D., Spelke, E. S. (2007). Core system in human cognition. Progress in Brain Research, 164, 257-264. doi: 10.1016/S0079-6123(07)64014-X

Klein, R. A., Ratliff, K. A., Vianello, M., Adams, R. B., Jr., Bahník, Š., Bernstein, M. J., ... Nosek, B. A. (2014). Investigating variation in replicability: A "many labs" replication 


\section{INFANTS' SOCIAL EVALUATIONS}

project. Social Psychology, 45(3), 142-152. doi: 10.1027/1864-9335/a000178.

Klein, R.A., Ratliff, K.A., Vianello, M., Adams Jr., R.B., Bahník, S., Bernstein, M.J., .. Nosek, B.A., 2014. Data from investigating variation in replicability: A "Many Labs" replication project. Journal of Open Psychology Data, 2(1), p.e4. doi: http://doi.org/10.5334/jopd.ad

Kohlberg, L. (1969). Stages in the development of moral thought and action. New York, NY:

Holt, Rinehart \& Winston.Kominsky, J. F. (2019). PyHab: Open-source real time infant gaze coding and stimulus presentation software. Infant Behavior \& Development, 54, 114-119.

Kruschke, J. K., \& Liddell, T. M. The Bayesian New Statistics: Hypothesis testing, estimation, meta-analysis, and planning from a Bayesian perspective. Psychonomic Bulletin and Review. doi: 10.3758/s13423-016-1221-4

Kvarven, A., Strømland, E., \& Johannesson, M. (2020). Comparing meta-analyses and preregistered multiple-laboratory replication projects. Nature Human Behaviour, 4(4), 423-434.

Liu, S., Brooks, N. B., \& Spelke, E. S. (2019). Origins of the concepts cause, cost, and goal in prereaching infants. Proceedings of the National Academy of Sciences, 116(36), 1774717752.

Loheide-Niesmann, L., de Lijster, J., Hall, R., van Bakel, H., \& Cima, M. (2020). Toddlers' preference for prosocial versus antisocial agents: No associations with empathy or attachment security. Social Development.

Lucca, K., Pospisil, J., \& Sommerville, J. A. (2018). Fairness informs social decision making in 


\section{INFANTS' SOCIAL EVALUATIONS}

infancy. PloS One, 13(2), e0192848.

Makel, M. C., Plucker, J. A., \& Hegarty, B. (2012). Replications in Psychology Research: How Often Do They Really Occur? Perspectives on Psychological Science: A Journal of the Association for Psychological Science, 7(6), 537-542.

Margoni, F., \& Shepperd, M. (2020). Changing the logic of replication: A case from infant studies. Infant Behavior and Development, 61C, 101483.

Margoni, F., \& Surian, L. (2018). Infants' evaluation of prosocial and antisocial agents: A metaanalysis. Developmental Psychology, 54(8), 1445-1455. http://dx.doi.org/10.1037/dev0000538

Marquardt, D. W. (1980). Comment: You should standardize the predictor variables in your regression models. Journal of the American Statistical Association, 75(369), 87-91.

Morey, R. D., Hoekstra, R., Rouder, J. N., Lee, M. D., \& Wagenmakers, E. J. (2016). The fallacy of placing confidence in confidence intervals. Psychonomic bulletin \& review, 23(1), 103-123. doi: 10.3758/s13423-015-0947-8

Nighbor, T., Kohn, C., Normand, M. \& Schlinger, H. (2017). Stability of infants' preference for prosocial others: Implications for research based on single-choice paradigms. PLoS One, 12(6), e0178818.

Oakes, L. M. (2017). Sample size, statistical power, and false conclusions in infant looking-time research. Infancy: The Official Journal of the International Society on Infant Studies, $22(4), 436-469$.

Open Science Collaboration, Nosek, B. A., Aarts, A. A., Anderson, C. J., Anderson, J. E., \& 


\section{INFANTS' SOCIAL EVALUATIONS}

Kappes, H. B. (2015). Estimating the reproducibility of psychological science. Science, Vol. 349, no. 6251, pp. aac4716. https://doi.org/10.1126/science.aac4716issn: 0036-8075

Peirce, J., Gray, J. R., Simpson, S., MacAskill, M., Höchenberger, R., Sogo, H., Kastman, E., \& Lindeløv, J. K. (2019). PsychoPy2: Experiments in behavior made easy. Behavior Research Methods, 51(1), 195-203. https://doi.org/10.3758/s13428-018-01193-y

Plummer, M., Best, N., Cowles, K., \& Vines, K. (2006). CODA: convergence diagnosis and output analysis for MCMC. R news, 6(1), 7-11.

Piaget, J. (1932). The moral judgment of the child. London, England: Kegan Paul, Trench, Trubner.

Salvadori, E., Blazsekova, T., Volein, A., Karap, Z., Tatone, D., Mascaro, O., \& Csibra, G. (2015). Probing the strength of infants' preference for helpers over hinderers: Two replication attempts of Hamlin and Wynn (2011). PloS one, 10(11), e0140570.

Scarf, D., Imuta, K., Colombo, M., \& Hayne, H. (2012). Social Evaluation or Simple Association? Simple Associations May Explain Moral Reasoning in Infants. PloS One, 7(8): e42698. doig: 10.1371/journal.pone.0042698

Scarf, D., Imuta, K., Colombo, M., \& Hayne, H. (2012). Golden rule or valence matching? Methodological problems in Hamlin et al. Proceedings of the National Academy of Sciences, 109(22) E1426. doi: 10.1073/pnas.1204123109

Schlingloff, L., Csibra, G. \& Tatone, D. (2020). Do 15-month-old infants prefer helpers? A replication of Hamlin et al. (2007). Royal Society Open Science, 7: 191795. https://doi.org/10.1098/rsos.191795 


\section{INFANTS' SOCIAL EVALUATIONS}

Scola, C., Holvoet, C., Arciszewski, T., \& Picard, D. (2015). Further evidence for infants' preference for prosocial over antisocial behaviors. Infancy, 20(6), 684-692.

Shimizu, Y., Senzaki, S., \& Uleman, J. S. (2018). The influence of maternal socialization on infants' social evaluation in two cultures. Infancy, 23(5), 748-766.

Stanley, D., \& Spence, J. (2014). Expectations for replications: are yours realistic? Perspectives on Psychological Science, 9, 305-318. doi: 10.1177/1745691614528518

Tasimi, A., \& Wynn, K. (2016). Costly rejection of wrongdoers by infants and children. Cognition, 151, 76-79.

Van de Vondervoort, J. W., \& Hamlin, J. K. (2017). Preschoolers' social and moral judgments of third-party helpers and hinderers align with infants' social evaluations. Journal of Experimental Child Psychology, 164, 136-151.

Vaporova, E. \& Zmyj, N. (2020). Social evaluation and imitation of prosocial and antisocial agents in infants, children, and adults. PLoS One, 15(9), e0235595.

Woo, B. M., Steckler, C. M., Le, D. T., \& Hamlin, J. K. (2017). Social evaluation of intentional, truly accidental, and negligently accidental helpers and harmers by 10 -month-old infants. Cognition, Vol. 168, pp. 154-163. doi: 10.1016/j.cognition.2017.06.029

Zwaan, R. A., Etz, A., Lucas, R. E., \& Donnellan, M. B. (2018). Making replication mainstream. Behavioral and Brain Sciences, 41, E120. doi: 10.1017/S0140525X17001972 


\section{Tables}

$\begin{array}{lll}\text { Lab Location } & \text { Mean age } & N \\ \text { University Name } & \text { XX } & \text { X } \\ \text { University Name } & \text { XX } & \text { X }\end{array}$

Table 1. Details about included labs.

\begin{tabular}{lccc}
\hline Parameter & Estimate & Est. Error & $95 \%$ CI \\
\hline Intercept & -0.10 & 0.09 & {$[-0.27,0.08]$} \\
condition & 0.57 & 0.09 & {$[0.39,0.75]$} \\
Z_age_days & -0.15 & 0.13 & {$[-0.41,0.12]$} \\
condition:z_age_days & 0.16 & 0.19 & {$[-0.20,0.53]$} \\
\hline
\end{tabular}

Table 2. Parameter estimates along with estimated error and 95\% Credible Intervals (CI) for those parameters for the full Bayesian model with informative priors, on simulated data.

\begin{tabular}{lccc}
\hline Parameter & Estimate & Est. Error & $95 \%$ CI \\
\hline Intercept & 0.12 & 0.14 & {$[-0.15,0.38]$} \\
condition & 0.71 & 0.24 & {$[0.25,1.19]$} \\
Z_age_days & -0.17 & 0.14 & {$[-0.43,0.11]$} \\
condition:z_age_days & 0.21 & 0.20 & {$[-0.18,0.61]$} \\
\hline
\end{tabular}

Table 3. Parameter estimates along with estimated error and 95\% Credible Intervals (CI) for those parameters for the full Bayesian model with non-informative priors, on simulated data. 


\section{Figures}

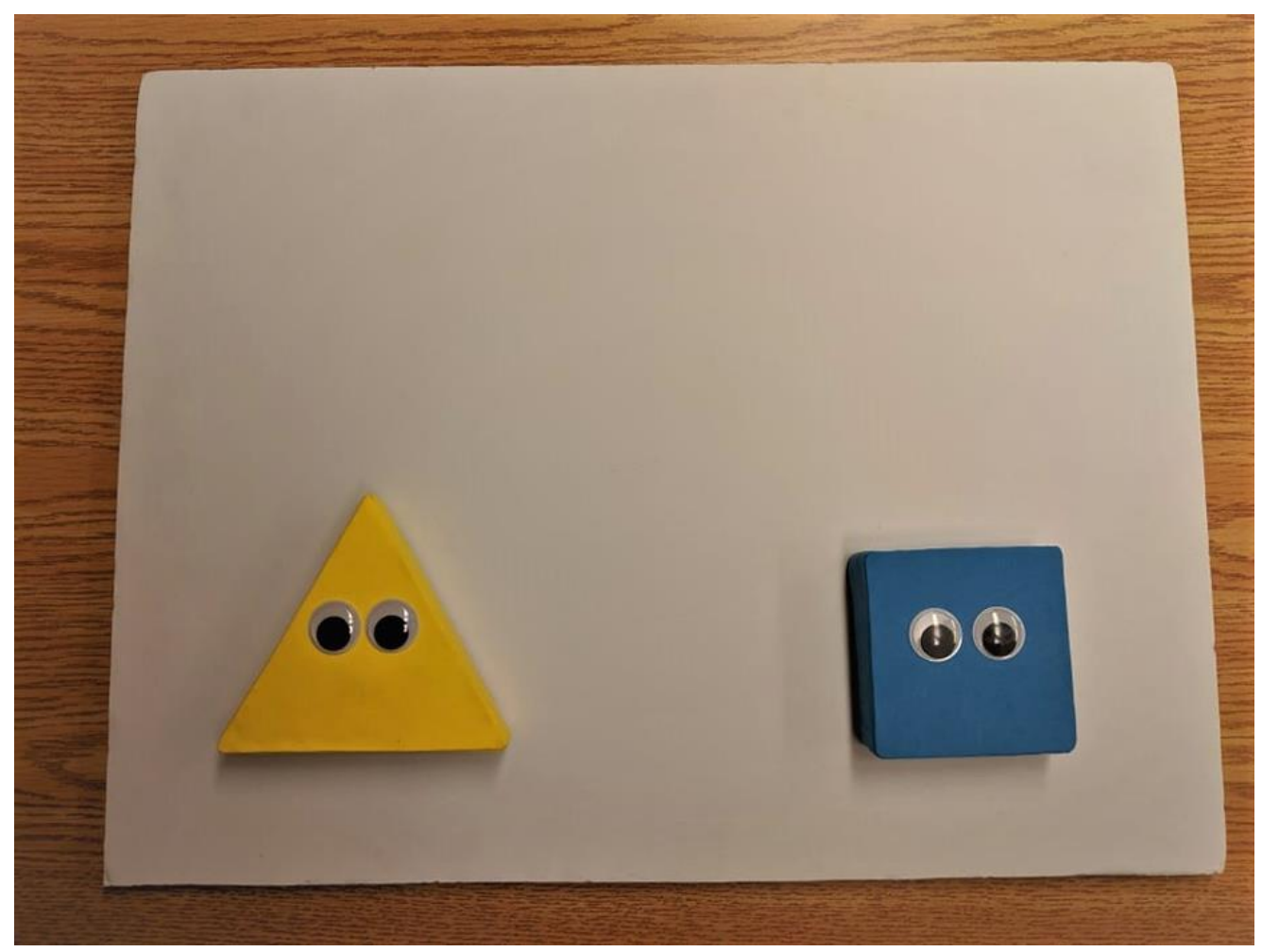

Figure 1. Choice phase foam 3-D stimuli presented on a white board. Board dimensions: $45 \mathrm{~cm} \mathrm{x}$ $60 \mathrm{~cm}$; Shape dimensions: $9.9 \mathrm{~cm}$ x $9.9 \mathrm{~cm}$ (blue square), $14.8 \mathrm{~cm}$ (base) x $13 \mathrm{~cm}$ (height; yellow triangle). 


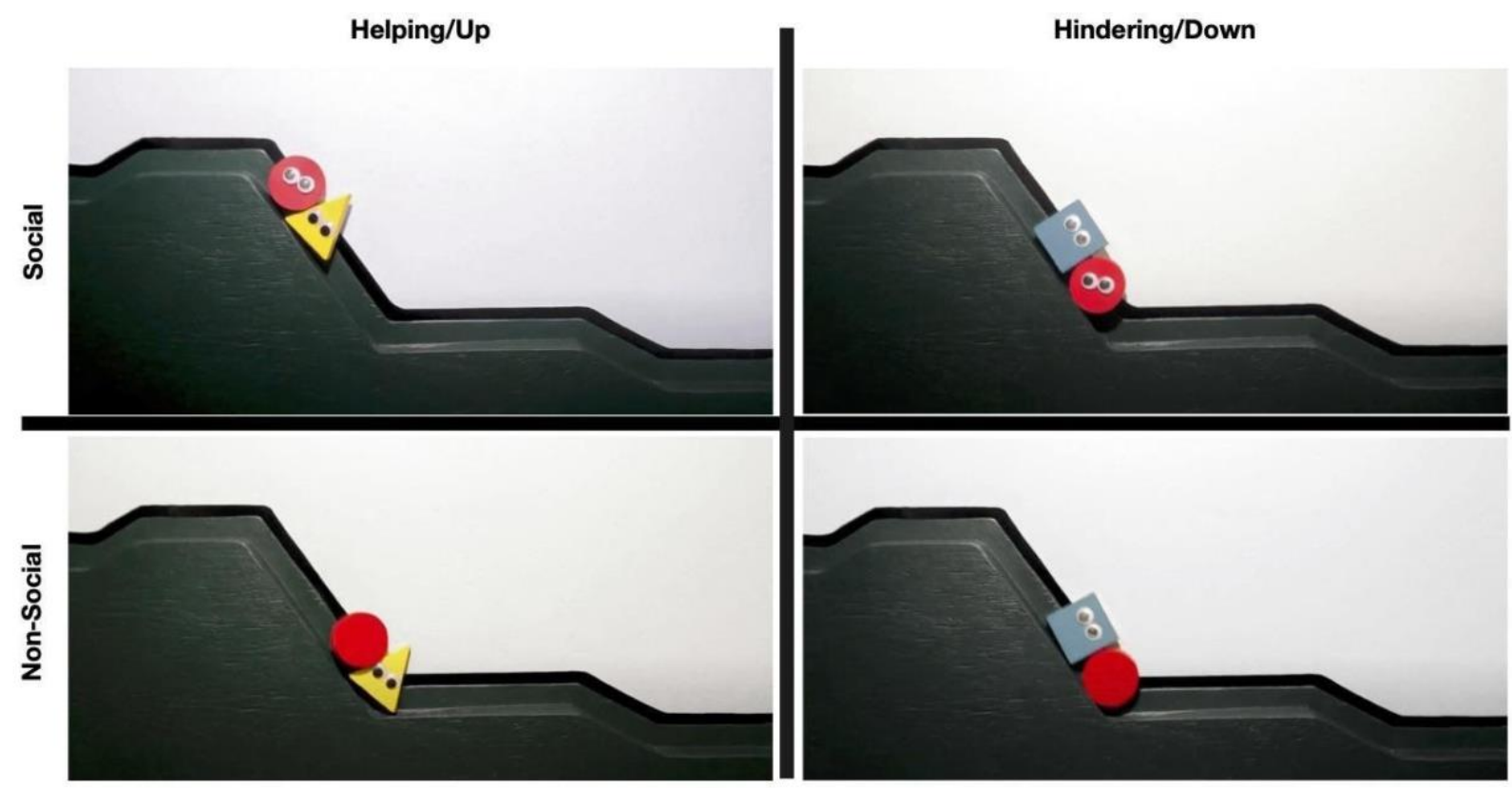

Figure 2. Screenshots of helping (top left) and hindering (top right) events in the Social Condition; pushing up actions (bottom left) and pushing down actions (bottom right) in the NonSocial Condition.

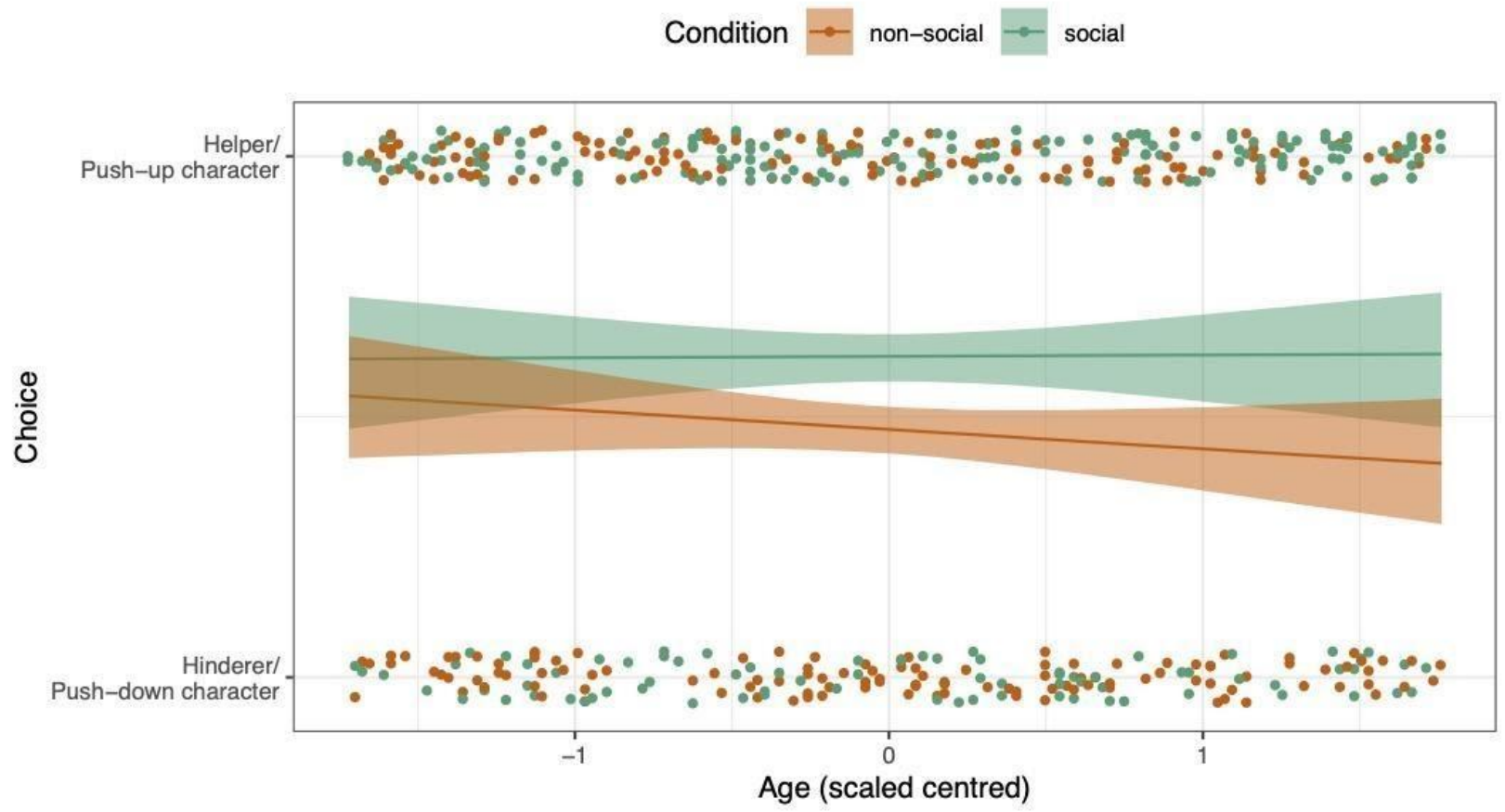

Figure 3. Probability of choosing the Helper/Push-up Character (over Hinderer/Push-down Character) across ages from simulated data. The smoothing line shows the predicted marginal effects from our Bayesian regression model along with their credible interval. Data are jittered slightly on the vertical axis to avoid overplotting. 


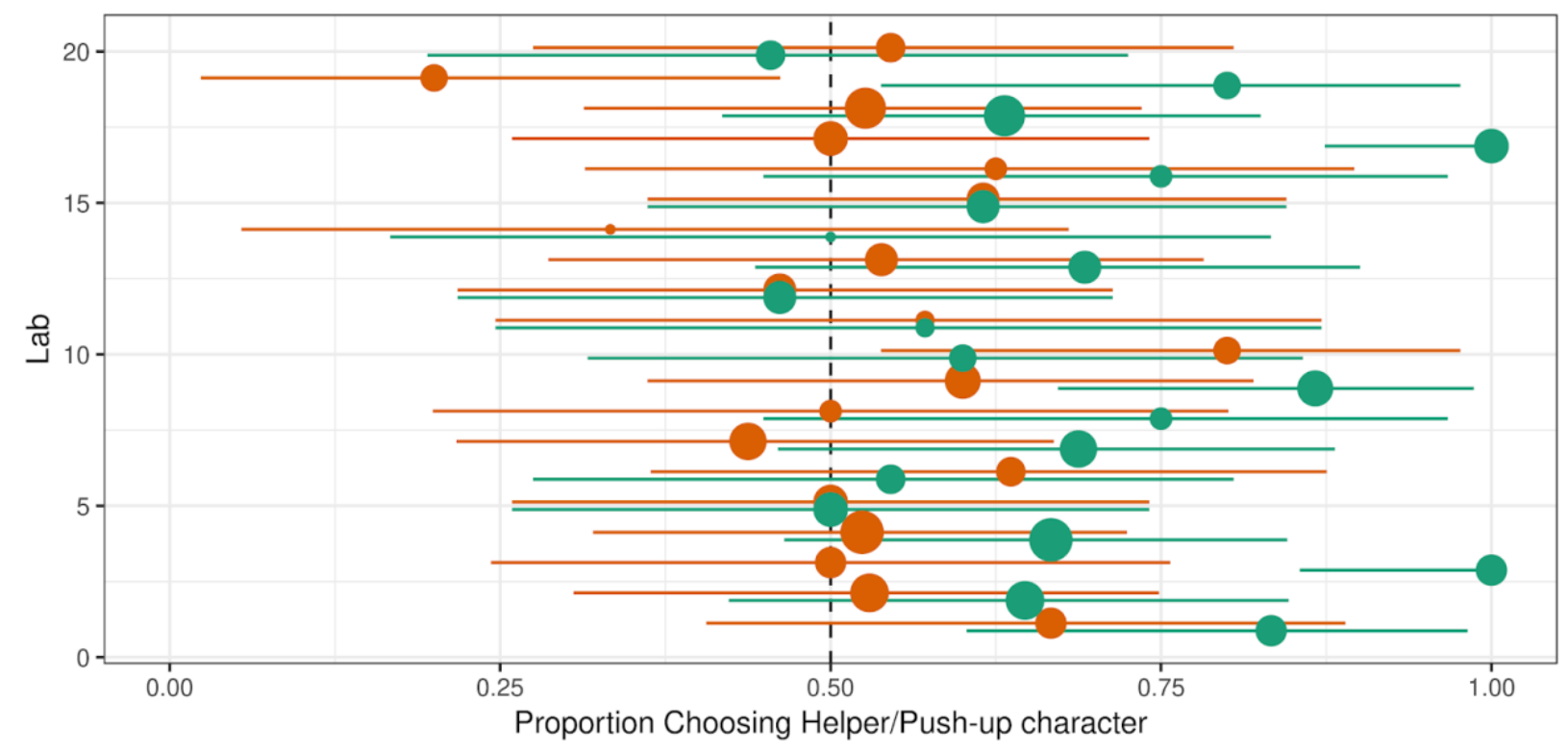

$N \cdot 6.0 \bigcirc 13.5 \bigcirc 21.0$ Condition $\phi$ non-social $\phi$ social

Figure 4. "Forest plot" of estimates for proportion of participants selecting the Helper/Push-up Character (over Hinderer/Push-down Character) for each contributing lab from simulated data. Dotted line represents chance performance. Error bars represent 95\% Bayesian Credible Intervals. 